\title{
Editoriale
}

\section{La denervazione renale transcatetere: il ruolo del nefrologo}

\section{F. Pieruzzi}

Università degli Studi di Milano Bicocca, Dipartimento di Scienza della Salute, Clinica Nefrologica, Azienda Ospedaliera S. Gerardo, Monza

RENAL DENERVATION AND NEPHROLOGIST'S ROLE

AвSTRACт. Recent clinical trials have shown that catheter based bilateral sympathetic renal denervation can significantly reduce blood pressure values in resistant hypertensive patients. In addition to this blood pressure lowering effect this procedure determines a significant reduction of the sympathetic activity, of insulin resistance and of cardio-renal target organ damage. Catheter based renal denervation is a safe procedure with very limited side effects. Although the clinical evidence obtained from recent clinical trials showing that renal denervation can significantly reduce blood pressure values in resistant hypertensive patients is solid, it is important to test this procedure in the real clinical setting. This is the principal aim of the Italian Registry which will collect data on the efficacy and safety of this procedure in the short and long term. However, the effects of the procedure on the renal function, proteinuria and sodium balance are still not well known in particular in the long term. These informations are required in order to extend the indications of the renal denervation in different pathological conditions characterized by elevated sympathetic nervous activity, and to establish the cost-effectiveness of this procedure as a potential alternative as compared to the traditional pharmacological treatments.

Key Words. Renal denervation, Sympathetic nerve traffic, Resistant hypertension, Renal function

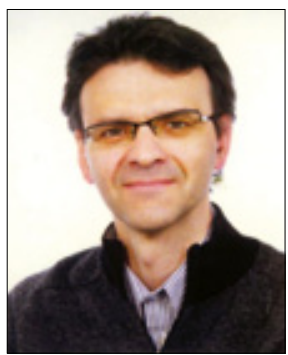

Studi clinici recenti hanno evidenziato che la procedura di denervazione renale bilaterale mediante catetere stimolatore in radiofrequenza è in grado di esercitare nell'ipertensione arteriosa resistente a tre o più farmaci antipertensivi una significativa riduzione pressoria di entità pari a 20-25 $\mathrm{mmHg}$ per la sistolica e $10-$ $20 \mathrm{mmHg}$ per la diastolica. All'efficacia antipertensiva, documentata nell'arco di tutti i 3 anni di osservazione sinora valutati da studi clinici prospettici, la metodica associa una significativa riduzione dell'ipertono simpatico, dell'insulino-resistenza e del danno d'organo cardiaco e renale (ipertrofia ventricolare sinistra e microalbuminuria), di frequente riscontro nelliperteso resistente alla terapia. La procedura, inoltre, si è rivelata sicura e pressoché priva di effetti collaterali. Infatti l'approccio percutaneo transcatetere, in grado di interrompere selettivamente le fibre nervose simpatiche renali, costituisce una soluzione meno invasiva, gravata da molti meno effetti collaterali rispetto agli storici trattamenti chirur- gici di simpaticectomia utilizzati per il trattamento dell'ipertensione arteriosa (1).

Tuttavia rimangono da verificare la sicurezza a lungo termine di questa procedura e i suoi effetti sulla morbilità e mortalità. Inoltre vi sono numerosi aspetti meritevoli di approfondimento e di discussione in particolare per gli aspetti più specificatamente nefrologici di questa innovativa e sicuramente interressante procedura.

Innanzitutto i dati della letteratura, che hanno permesso l'introduzione della tecnica di denervazione renale transcatetere nella pratica clinica, al momento si riferiscono quasi esclusivamente a pazienti con ipertensione resistente e sono stati pubblicati dallo stesso gruppo di lavoro. In entrambi gli studi Symplicity HTN-1 e $2(2,3)$ una certa percentuale di pazienti affetti da ipertensione arteriosa resistente (rispettivamente il 13 e il 16\%) è risultata non responsiva al trattamento di denervazione renale percutanea. Si può ipotizzare che in questi pazienti o la metodica non è risultata efficace nel determinare un'ablazione terapeutica dei nervi renali, oppure le fibre nervose simpatiche renali (afferenti ed efferenti) non rivestivano un ruolo cruciale nella patogenesi dell'i- 
pertensione di questi soggetti. Un recentissimo lavoro pubblicato su Hypertension per la prima volta afferma che in un gruppo seppur limitato di ipertesi resistenti non selezionati, non è stato possibile dimostrare un efficace calo pressorio ed una concomitante riduzione del traffico nervoso simpatico periferico (4). Gli autori spiegano tale fenomeno affermando che i pazienti "non responders" potrebbero essere più numerosi rispetto a quanto previsto dagli studi pubblicati in precedenza ed auspicano la necessità di poter identificare pazienti che potrebbero beneficiare del trattamento ablativo. Per identificare i soggetti potenzialmente più responsivi alla procedura, potrebbe essere utile introdurre nei criteri di selezione dei pazienti ipertesi resistenti da sottoporre a denervazione renale, la valutazione del traffico nervoso periferico a livello muscolare (MSNA), in quanto possibile predittore della risposta pressoria al trattamento con radiofrequenze. Sarebbe interessante cercare di caratterizzare ulteriormente il profilo del sistema nervoso simpatico pre- e post-procedura di questi pazienti (con la microneurografia o con altre metodiche) e capire se la mancata risposta al trattamento sia dovuta a una mancata interruzione delle fibre nervose renali oppure sia legata a una tipologia d'ipertensione la cui eziopatogenesi non risulti influenzata dalle fibre nervose renali afferenti e/o efferenti. In ogni caso, non sono al momento disponibili nell'uomo procedure che possano documentare in modo diretto l'attività nervosa simpatica renale e quindi l'avvenuta interruzione delle fibre nervose renali.

In aggiunta ad oggi non esistono tecniche dirette facilmente applicabili nell'uomo per stabilire, nell'immediato, se la denervazione renale sia avvenuta o meno con successo e soprattutto per quantificare l'entità dell'ablazione (totale, parziale, unilaterale?). Tuttavia, a dimostrazione dell'efficacia della procedura, nello studio Symplicity HTN-1 (2) lo spillover renale di noradrenalina, seppur dosato solo in 10 pazienti, si era ridotto del 47\% a 1 mese dal trattamento. Un studio non facilmente riproducibile su ampie casistiche appena pubblicato ha validato l'efficacia della denervazione transcatere valutando la mancata risposta ipertensiva a una stimolazione ad alta frequenza dell'arteria renale post-procedura (5). Un'altra problematica aperta dalla denervazione renale è la possibilità che, nel lungo periodo, possa avvenire una reinnervazione funzionale, annullando gli effetti ottenuti grazie alla procedura. A tal proposito bisogna ricordare che in letteratura non vi sono evidenze di reinnervazione afferente. Per quanto riguarda le efferenze, invece, sono state segnalate, in pazienti sottoposti a trapianto renale, delle evidenze istologiche di reinnervazione (6), non seguite, tuttavia, da evidenze di reinnervazione funzionale (7). Ad ogni modo, le evidenze ottenute fino ad ora, in particolare nella prosecuzione del Symplicity HTN-1 (2), in cui nei pazienti in follow-up fino a 24 mesi si è mantenuta la consistente e significativa riduzione dei valori di pressione arteriosa, escluderebbe la possibilità di reinnervazione funzionale. Gli autori inoltre affermano che nel caso in cui si dovesse osservare una reinnervazione funzionale a distanza di tempo, date le caratteristiche di ridotta invasività della procedura, al momento non sembrano esserci controindicazioni alla possibilità di ripetere la procedura.

Sebbene siano stati pubblicati pochi dati che riportino problemi tecnici o complicanze legate alla procedura, è probabile che con l'incremento della casistica in diverse realtà cliniche vi sia la necessità di monitorare anche questi aspetti. Un caso clinico recentemente apparso su Lancet segnalava l'insorgenza di un significativo rialzo pressorio a distanza di alcuni mesi da una efficace procedura di denervazione renale con iniziale riduzione dei valori pressori, legata alla formazione di una stenosi di un'arteria renale (8). Gli autori suggeriscono un'accurata valutazione ecocolorDoppler periodica nel follow-up post-denervazione. In uno studio sperimentale istopatologico su modello animale, Steigerwald (9) ha dimostrato che la termoablazione delle arterie renali determina acutamente un transitorio danno endoteliale potenzialmente trombogenico meritevole di ulteriori approfondimenti nella pratica clinica.

Numerosi studi di ricerca sperimentale, e le conoscenze fisiopatologiche del ruolo dell'attivazione del sistema nervoso simpatico in altri contesti patologici indipendenti dall'ipertensione arteriosa resistente, rappresentano un'importante premessa all'estensione delle indicazioni cliniche del trattamento con denervazione renale transcatetere. Probabilmente in un futuro molto più vicino di quanto ipotizzabile il nefrologo verrà coinvolto in maggior misura in questo ambito dato che la necessità di incrementare le conoscenze degli effetti della denervazione renale è proprio rivolta agli effetti della procedura sulla funzione renale.

Un'elevata MSNA basale giustificherebbe l'estensione del campo di applicazione della denervazione renale a tutte quelle forme di ipertensione caratterizzate da iperattività simpatica (10) e a quelle condizioni cliniche, associate o meno ad ipertensione arteriosa, in cui è presente un'aumentata MSNA. Liperattivazione cronica del sistema nervoso simpatico costituisce, infatti, il denominatore comune di numerose patologie: le nefropatie croniche con o senza insufficienza renale, lo scompenso cardiaco, i disturbi del sonno, l'obesità, la sindrome metabolica e il rene policistico.

Studi su modelli sperimentali con insufficienza renale cronica, hanno dimostrato che la somministrazione di 
moxonidina, un farmaco agonista alfa-2 adrenergico, a dosi non antipertensive, è in grado di causare una riduzione significativa della proteinuria (11). Questo reperto suggerisce l'esistenza di un nesso tra l'attività del sistema nervoso simpatico renale e la proteinuria, a prescindere dalla pressione arteriosa. Nell'uomo, invece, non esistono evidenze in questo senso anche se è lecito supporre che la denervazione possa avere un effetto protettivo sulla funzione renale. Non sono tuttavia chiare nell'uomo, le ripercussioni della denervazione sull'emodinamica renale, per cui diventa difficile comprendere se l'effetto antiproteinurico indotto dalla denervazione renale sia semplicemente legato alla riduzione della pressione arteriosa, o intervenga anche una riduzione dell'influenza delle efferenze simpatiche renali sul rene stesso.

Un recentissimo studio (12) ha dimostrato, a 3 e 6 mesi dalla denervazione renale transcatetere, una lieve riduzione degli indici di resistenza renale, in 88 soggetti ipertesi resistenti rispetto ai controlli. Questa prima dimostrazione suggerisce che la denervazione renale potrebbe avere un effetto benefico anche sull'emodinamica renale, verosimilmente mediato dalla riduzione della vasocostrizione simpatica renale riflessa.

Nei pazienti nefropatici l'ipertensione arteriosa è un riscontro molto comune e peggiora con l'instaurarsi e il progredire dell'insufficienza renale, infatti circa l' $85 \%$ dei pazienti affetti da insufficienza renale terminale è iperteso (13). È ampiamente accettato che l'ipertensione contribuisce al progressivo deterioramento della funzione renale e inoltre rende ragione dell'elevata incidenza di eventi cardiovascolari che costituiscono la principale causa di morte di questi pazienti. Infatti, nella pratica clinica quotidiana, spesso risulta difficile correggere farmacologicamente l'ipertensione associata ad insufficienza renale, verosimilmente perché essa riconosce diversi fattori patogenetici. In particolare, tra $\mathrm{i}$ fattori coinvolti nella genesi dellipertensione in pazienti nefropatici, liperattivazione simpatica cronica sembra rivestire un ruolo fondamentale sin dagli stadi iniziali di insufficienza renale, come recentemente confermato da uno studio di Grassi e collaboratori (14). Queste considerazioni fanno del malato nefropatico iperteso resistente, un candidato ideale da sottoporre a denervazione renale. Recentemente è stato pubblicato uno studio pilota condotto su 15 pazienti affetti da insufficienza renale moderata-severa (eGFR medio $=31 \mathrm{~mL} / \mathrm{min}$ ) e ipertensione resistente (15). In un sottogruppo di pazienti (6 su 15) è stata utilizzata una tecnica angiografica a $\mathrm{CO}_{2}$ per minimizzare l'esposizione a mezzo di contrasto iodato (50 mL vs $80 \mathrm{~mL}$ circa). La denervazione renale bilaterale, in questo studio pilota, si è dimostrata efficace nel ridurre la pressione arteriosa in assenza di alterazioni dell'eGFR nel breve e nel lungo termine inoltre gli autori hanno osservato una riduzione (seppur statisticamente non significativa vista la scarsa numerosità del campione oggetto di studio) dei valori di proteinuria delle 24 ore e di UACR. Nessuna differenza è stata riscontrata, in termini di alterazioni della funzione renale, tra il gruppo trattato con angiografia standard e il sottogruppo trattato con angiografia a $\mathrm{CO}_{2}$. Dai risultati di questo primo piccolo studio si può dedurre che apparentemente la denervazione renale non solo non peggiorerebbe l'insufficienza renale pre-esistente, ma potrebbe, nel lungo termine, rallentare la progressione del danno renale riducendo sia pressione arteriosa che escrezione urinaria di proteine, note per essere le principali cause di deterioramento della funzione renale. Tali potenzialità andranno, verificate in studi successivi. Non bisogna, tuttavia, sottovalutare i rischi connessi all'utilizzo del mezzo di contrasto iodato, soprattutto nella prospettiva di allargare l'utilizzo di questa procedura a una popolazione più ampia.

All'inizio degli anni '90 Converse e collaboratori (16) hanno dimostrato che la nefrectomia bilaterale in pazienti emodializzati ipertesi, oltre a ridurre la pressione arteriosa, correggeva l'aumentato tono simpatico presente in questi pazienti. La denervazione renale percutanea, per definizione meno invasiva dell'asportazione chirurgica dei reni nativi, potrebbe pertanto essere applicata con successo anche alla popolazione di emodializzati che, come già accennato, tra i pazienti nefropatici sono i più colpiti da ipertensione arteriosa. Al momento in letteratura è disponibile solo un case report di un soggetto emodializzato di 39 anni affetto da ipertensione resistente (17), in cui la denervazione renale percutanea si è dimostrata efficace e sicura nel ridurre la pressione arteriosa: in particolare non sono state osservate alterazioni della diuresi residua di $500 \mathrm{~mL}$.

Analogamente a quanto osservato nei pazienti in emodialisi, anche nei pazienti trapiantati ipertesi la normalizzazione dell'MSNA si ottiene solo dopo nefrectomia bilaterale dei reni nativi (18), pertanto la denervazione renale potrebbe avere un ruolo anche nel trattamento dell'ipertensione dopo trapianto renale.

Sempre in campo nefrologico un'aumentata MSNA è stata documentata anche in pazienti ipertesi affetti da rene policistico autosomico dominante (19) con funzione renale normale. Un caso clinico pubblicato recentemente (20) ha descritto l'applicazione della denervazione renale trans-catetere in una donna di 58 anni affetta da ipertensione resistente e rene policistico autosomico dominante con funzione renale conservata: la procedura si è dimostrata, anche in questa condizione clinica, 
sicura ed efficace nel ridurre i valori pressori e collateralmente è stata segnalata anche una risoluzione del dolore lombare cronico secondario alla presenza delle cisti renali.

L'iperattivazione dei nervi renali afferenti ed efferenti esercita un ruolo cruciale, come già accennato, anche nella patogenesi dello scompenso cardiaco. I segnali nervosi afferenti a partenza dal cuore e dai vasi sanguigni provocano un'aumentata attività nervosa simpatica renale efferente riflessa con incremento della secrezione di renina, della ritenzione di sodio e riduzione del flusso plasmatico renale: tre condizioni caratteristiche della sindrome cardiorenale. Uno studio pilota per valutare la sicurezza della denervazione renale in una ristretta popolazione di pazienti con scompenso cardiaco (21) ha posto le basi per il trial clinico, Symplicity HF (http://clinicaltrials.gov/ct2/show/ study/NCT01392196), attualmente in fase di reclutamento, che si propone di sottoporre a denervazione renale 40 pazienti affetti da scompenso cardiaco (classe NYHA II-III, con FE minore del 40\%) e concomitante insufficienza renale (eGFR 30-75 $\mathrm{mL} / \mathrm{min} / 1.73 \mathrm{~m}^{\wedge} 2$ ). L'obiettivo dei ricercatori è valutare il profilo di sicurezza nello scompenso e gli effetti sulla funzione renale e cardiaca. Questo studio potrebbe aprire delle prospettive terapeutiche non solo nello scompenso cardiaco ma anche nella sindrome cardiorenale (22).

I numerosi risvolti terapeutici correlati alla riduzione dell'attivazione simpatica dopo denervazione renale, osservati in diverse patologie caratterizzate da ipertono simpatico e ipertensione arteriosa, potrebbero apportare un beneficio anche in termini prognostici ai pazienti trattati. È noto infatti che, oltre allo stato ipertensivo stesso, anche un'iperattivazione adreneregica cronica contribuisce allo sviluppo e alla progressione del danno d'organo periferico cardiaco, vascolare e renale.

Attualmente è disponibile nella pratica clinica un solo elettrocatetere progettato specificamente per la denervazione renale. Tuttavia sono numerose le novità tecnologiche che sono in corso di sperimentazione o di validazione, che potrebbero permettere una riduzione della durata della procedura e una riduzione dell'esposizione al mezzo di contrasto, particolarmente interessante nei pazienti con insufficienza renale cronica avanzata, a parità di efficacia di erogazione ablativa (23).

$\mathrm{E}$ attualmente in fase di studio (http://clinicaltrials. gov/ct2/show/study/ NCT01438229) un nuovo catetere a radiofrequenze, il catetere tipo basket, dotato di più elettrodi che, mediante un'unica erogazione di energia, potrebbero determinare un'ablazione più estesa e più rapida delle fibre nervose renali.

Inoltre sono in corso di sperimentazione nuove tecni- che di denervazione renale come l'applicazione locale di farmaci neurotossici, la crioablazione e la denervazione indotta da ultrasuoni (24) che potrebbero chiarire se la mancata responsività al trattamento di termoablazione dipenda o meno da un limite della metodica di ablazione utilizzata.

I 3 casi presentati da Arienzo e collaboratori in questo numero di $T N \& D$ sono la dimostrazione del riscontro nella realtà clinica dell'impiego della denervazione renale in soggetti particolarmente complicati, con valori pressori e numero di farmaci antipertensivi molto superiori a quella degli studi controllati e con elevata morbilità. Anche in questi soggetti è stato possibile ottenere degli effetti favorevoli anche se non sono stati raggiunti cali pressori paragonabili ai dati presenti in letteratura.

Nonostante queste interessanti prospettive future, anche nel lavoro di Arienzo viene giustamente sollevato il problema dei costi di questa procedura, viste le note difficoltà economiche nell'ambito del SSN in questo particolare contesto storico, e alla luce della complessità oggettiva nel prevedere un risparmio dovuto allimpiego della tecnica transcatetere nei confronti del tradizionale approccio farmacologico nel trattamento dell'ipertensione arteriosa. Certamente dati più consistenti sulla reale riduzione del numero di farmaci utilizzati e di un'eventuale riduzione della morbilità e mortalità potrebbero chiarire questo delicato aspetto che tuttavia potrebbe dar luogo a un impiego su vasta scala di questa procedura (25).

Il punto cruciale quindi rimane la necessità di ampliare la casistica e in particolare di verificare con metodo e rigore l'attività clinica al di fuori degli studi clinici controllati. A tale scopo alcune società nazionali dell'ipertensione arteriosa europee stanno emanando raccomandazioni o documenti di "expert consensus", al fine di regolamentare la selezione dei pazienti, di definire un comune approccio tecnico della denervazione renale, e di proporre un percorso di gestione clinica specifico per i candidati alla procedura (26-28). In questi documenti si sottolinea la necessità, dopo aver correttamente identificato $i$ candidati alla procedura di denervazione renale, di affidarli ai Centri Nazionali di Eccellenza o ai Centri di Riferimento con documentata esperienza procedurale. In questo contesto viene anche raccomandato l'impiego di un registro prospettico per fornire dati oggettivi e applicabili alla realtà clinica. Questa esigenza è sentita anche dall'azienda che produce il catetere attualmente in commercio, che ha istituito il Registro Internazionale Symplicity ${ }^{\circledR}(\mathrm{http}: / /$ clinicaltrials.gov/ ct2/show/NCT01534299) che raccoglierà e analizzerà i dati di almeno 5000 pazienti sotto- 
posti a denervazione renale, in più di 200 Centri dislocati in tutto il mondo. Nel nostro Paese è attualmente in corso il Registro Prospettico Italiano sulla denervazione renale transcatetere, promosso dalla Società Italiana dell'Ipertensione Arteriosa, che si propone di raccogliere informazioni relative all'efficacia e alla sicurezza di impiego a breve e a lungo termine della procedura nell'ambito della pratica clinica quotidiana. A questo scopo vengono raccolti i casi di pazienti sottoposti alla procedura da parte di diversi Centri Ospedalieri dislocati nel territorio Nazionale. L'esigenza di istituire un registro nazionale sulla denervazione renale nasce dal fatto che sebbene i dati della letteratura sull'efficacia della denervazione renale transcatetere siano abbastanza solidi, solo un'analisi dell'utilizzo in campo clinico di questa metodica potrà fornire un reale parametro di giudizio sulle modalità di esecuzione della procedure nelle diverse realtà cliniche sul territorio nazionale. In particolare, aderendo al registro, tutti gli operatori si dovrebbero attenere alle stesse procedure, follow-up e raccolta dei parametri, in modo da poter paragonare $\mathrm{i}$ dati in modo omogeneo. Una precisa e attenta gestione del registro da parte di personale qualificato e dedicato sia negli aspetti clinici che di raccolta dati è mandatoria per rendere il registro uno strumento utile per la comunità scientifica.

\section{Conclusioni}

Il ruolo della denervazione renale nel trattamento dell'pertensione arteriosa resistente è andato consolidandosi in questi ultimi anni, soprattutto a livello internazionale, grazie principalmente agli studi Symplicity HTN-1 ed HTN-2. E ora in corso lo studio Symplicity HTN-3 (29), uno studio randomizzato controllato in singolo cieco, che fornirà ulteriori dati sul profilo di efficacia e di sicurezza della metodica.

L'innovazione apportata da questo intervento consiste nell'agire in un pathway fisiopatologico a monte rispetto a molti dei comuni trattamenti farmacologici disponibili per l'ipertensione arteriosa, fornendo numerose ed interessanti prospettive di studio e trattamento. In aggiunta all'effetto sulla pressione arteriosa, infatti, sono emersi, grazie ai recenti studi effettuati sulla popolazione dei pazienti denervati, numerosi effetti positivi anche in altre patologie.

Pertanto la denervazione renale potrebbe costituire un trattamento sicuro che consente, con un'unica seduta di trattamento, di ridurre gli effetti sfavorevoli dell'attivazione nervosa simpatica, con molteplici conseguenze positive a livello cardiovascolare, nefrolo- gico, metabolico, che potrebbero determinare un miglioramento della qualità di vita e della sopravvivenza dei pazienti trattati.

\section{Riassunto}

Studi clinici recenti hanno dimostrato che la denervazione renale bilaterale con catetere a radiofrequenza riduce significativamente i valori pressori nell'ipertensione resistente. All'efficacia antipertensiva si associa una significativa riduzione dell'ipertono simpatico, dell'insulino-resistenza e del danno d'organo cardio-renale. La procedura si è rivelata pressoché priva di effetti collaterali.

Sebbene i dati della letteratura sull'efficacia antipertensiva della denervazione renale transcatetere siano solidi, solo un'analisi dell'utilizzo in campo clinico di questa metodica potrà fornire un reale parametro di giudizio sulle modalità di esecuzione della procedure nelle diverse realtà cliniche sul territorio nazionale. Per questo scopo è stato istituito il Registro Prospettico Italiano che raccoglie dati sull'efficacia e la sicurezza della denervazione renale a breve e a lungo termine.

Tuttavia non sono ancora noti gli effetti della procedura sulla funzione renale, la proteinuria e il bilancio del sodio, in particolare nel lungo termine. Queste conoscenze potranno permettere di estendere le indicazioni attuali della procedura anche in altre condizioni patologiche caratterizzate da ipertono simpatico e di valutare l'impatto del costo-beneficio della denervazione renale come un potenziale trattamento alternativo alle tradizionali strategie farmacologiche.

Parole Chiave. Denervazione renale, Funzione renale, Ipertensione resistente, Sistema nervoso simpatico

\author{
Indirizzo degli Autori: \\ Dr. Federico Pieruzzi \\ Università degli Studi di Milano Bicocca \\ Dipartimento di Scienza della Salute \\ Clinica Nefrologica \\ AO S Gerardo \\ Via Pergolesi 33 \\ Monza 20900 \\ federico.pieruzzi@unimib.it
}




\section{Bibliografia}

1. Krum H, Schlaid M, Whitbourn R, Sobotka PA, Sadowski $\mathrm{J}$, Bartus K, et al. Catheter-based renal sympathetic denervation for resistant hypertension: a multicenter safety and proof-of principle cohort study. Lancet 2009; 373: 1275-81.

2. Simplicity HTN-1 Investigators 2011. Catheter-based renal sympathetic denervation for resistant hypertension: durability of blood pressure reduction out to 24 months. Hypertension 2011; 57: 911-7.

3. Simplicity HTN-2 Investigators, Esler MD, Krum H, Sobotka PA, Schlaich MP, Schmieder RF, et al. Renal sympathetic denervation in patients with treatment resistant hypertension (The Simplicity THN-2 trial): a randomised control trial. Lancet 2010; 376: 1903-9.

4. Brinkmann J, Heusser K, Scmidt BM, Menne J, Klein G, Bauersachs J, Haller H, Sweep FC, Dietdrich A, Jordan J, Tank J. Catheter- based renal nerve ablation and centrally generated sympathetic activity in difficult-to-control hypertensive patients. Prospective case series. Hypertension 2012; Epub Oct 8.

5. Pokushalov E, Alexander Romanov A, Corbucci G, Artyomenko S, Baranova V, Turov A, Shirokova N, Karaskov A, Mittal S, Steinberg JS. A Randomized Comparison of Pulmonary Vein Isolation With Versus Without Concomitant Renal Artery Denervation in Patients With Refractory Symptomatic Atrial Fibrillation and Resistant Hypertension. J Am Coll Cardiol 2012; 60: 1163-70.

6. Gadzar AF, Dammin GJ. Neural degeneration and regeneration in human renal transplants. N Engl J Med 1970; 283(5): 222-4.

7. Hansen JM, Abildgaard U, Fogh-Andersen N, Kanstrup IL, Bratholm P, Plum I, Strandgaard S. The transplanted human kidney does not achieve functional reinnervation. Clin Sci (Lond) 1994; 87(1): 13-20.

8. Vonend O, Antoch G, Rump LC, Blondin D. Secondary rise in blood pressure after renal denervation. Lancet 2012; 380: 778.

9. Steigerwald K, Titovaa A, Mallea C, Kennerknechta C, Jileka C, Hausleitera J, Nahrigb JM, Laugwitza KL, Joner M. Morphological assessment of renal arteries after radiofrequency catheter-based sympathetic denervation in a porcine model. J Hypertens 2012; 30: 2230-9.

10. Grassi G. Assessment of sympathetic cardiovascular drive in human hypertension: achievements and perspectives. Hypertension 2009;54:690-7.

11. Amann K, Rump LC, Simonaviciene A, Oberhauser V, Wessels S, Orth SR, Gross ML, Koch A, Bielenberg GW, Van Kats JP, Ehmke H, Mall G, Ritz E. Effects of low dose sympathetic inhibition on glomerulosclerosis and albuminuria in subtotally nephrectomized rats. J Am Soc
Nephrol 2000; 11(8): 1469-78.

12. Mahfoud F, Cremers B, Janker J, et al. Renal hemodynamics and renal function after catheter-based renal sympathetic denervation in patients with resistant hypertension. Hypertension 2012; 60: 419-24.

13. Campese VM, Ku E, Park J. Sympathetic renal innervation and resistant hypertension. Int J Hypertens 2011. doi: 10.4061/2011/814354.

14. Grassi G, Quarti-Trevano F, Seravalle G, Arenare F, Volpe M, Furiani S, Dell'Oro R, Mancia G. Early sympathetic activation in the initial clinical stages of chronic renal failure. Hypertension 2011; 57(4): 846-51.

15. Hering D, Mahfoud F, Walton AS, et al. Renal Denervation in Moderate to Severe CKD. J Am Soc Nephrol 2012; 23: $1250-7$.

16. Converse RL Jr, Jacobsen TN, Toto RD, Jost CM, Cosentino F, Fouad-Tarazi F, Victor RG. Sympathetic overactivity in patients with chronic renal failure. N Engl J Med 1992; 327 (27):1912-8.

17. Di Daniele et al. Renal sympathetic nerve ablation for the treatment of difficult-to control or refractory hypertension in a haemodialysis patient. Nephrol Dial Transplant 2012); 27: 1689-90.

18. Hausberg M, Kosch M, Harmelink P, et al. Sympathetic nerve activity in end-stage renal disease. Circulation 2002; 106:1974-9.

19. Klein IH, Ligtenberg G, Oey PL, Koomans HA, Blankestijn PJ. Sympathetic activity is increased in polycystic kidney disease and is associated with hypertension. J Am Soc Nephrol 2001; 12(11): 2427-33.

20. Shetty SV, Roberts TJ, Schlaich MP. Percutaneous transluminal renal denervation: a potential treatment option for polycystic kidney disease-related pain? Int J Cardiol 2012, doi:10.1016/j.ijcard.2012.05.114.

21. Davies JE, Manisty CH, Petraco R, Barron AJ, Unsworth B, Mayet J, Hamady M, Hughes AD, Sever PS, Sobotka PA, Francis DP. First-in-man safety evaluation of renal denervation for chronic systolic heart failure: Primary outcome from REACH-Pilot study. Int J Cardiol 2012. Epub Sep 29.

22. Sobotka PA, Krum H, Bohm M, Francis DP, Schaich MP. The role of renal denervation in the treatment of heart failure. Curr Cardiol Rep 2012; 14: 285-92.

23. Lewalter T, Brodherr T. Three-Dimensional Guided Renal Denervation to Treat Drug-Resistant Arterial Hypertension in a Patient With Renal Insufficiency Hypertension, published online August 20, 2012.

24. Mabin T, Sapoval M, Cabane V, Stemmett J, Iyer M. First experience with endovascular ultrasound renal denervation for the treatment of resistant hypertension. Euro Intervention 2012; 8(1): 57-61.

25. Geisler BP, Egan BM, Cohen JT, Garner AM, Akehurst 
RL, Esler MD, Pietzsch JB. Cost-Effectiveness and Clinical Effectiveness of Catheter-Based Renal Denervation for Resistant Hypertension. J Am Coll Cardiol 2012; 60:1271-7.

26. Schmieder RE, Redon J, Grassi G, Kjeldsen S, Mancia G, Narkiewicz K, et al. ESH Position Paper: renal denervation - an interventional therapy of resistant hypertension. J Hypertens 2012; 30(5): 837-41.

27. Volpe M, Agabiti-Rosei E, Ambrosioni E, Cottone S, Cuspidi C, Borghi C, De Luca N, Fallo F, Ferri C, Mancia G, Morganti A, Muiesan ML, Sarzani R, Sechi L, Tocci $\mathrm{G}$, Virdis A. Denervazione delle arterie renali nel trattamento dell'ipertensione arteriosa resistente: definizione della patologia, selezione dei pazienti e descrizione della procedura. Documento di Indirizzo 2012 della SIIA. Ipertensione e prevenzione cardiovascolare 2012;19: 56-65.

28. Pathak A, Girerd X, Azizi M, Benamer H, Halimi JM, Lantelme P, Lefèvre T, Sapoval M; French Society of Hypertension; French Society of Cardiology; Working Group on Atheroma, Interventional Cardiology; French Society of Radiology. Expert consensus: renal denervation for the treatment of arterial hypertension. Arch Cardiovasc Dis 2012; 105(6-7): 386-93.

29. Kandzari DE, Bhatt DL, Sobotka PA, et al. Catheter-Based Renal Denervation for Resistant Hypertension: Rationale and Design of the SYMPLICITY HTN-3 Trial. Clin Cardiol 2012; 35: 528-35. 